\title{
Evolución del Contenido de Azufre en la Gasolina Comercializada en Galicia (España) como Consecuencia de la Aplicación del Real Decreto 1700/2003
}

\author{
Coro Fernández-Feal ${ }^{*}$, Luisa Fernández-Feal, Paola Escudero y José R. Pérez-Prado \\ Universidad de A Coruña, Centro de Investigaciones Tecnológicas, Laboratorio de Combustibles, \\ Campus de Esteiro s/n 15403, Ferrol-España (e-mail: combust@cdf.udc.es)
}

*autor a quien debe ser dirigida la correspondencia

\section{Resumen}

El objetivo de este estudio fue determinar la cantidad de azufre contenida en las gasolinas comercializadas en Galicia antes y después del 1 de enero de 2005, fecha establecida por ley, según Real Decreto 1700/2003, para la disminución del contenido de azufre en gasolinas. Esto se realizó con el fin de verificar el cumplimiento de la legislación vigente a partir de esa fecha: 150 $\mathrm{mg} / \mathrm{kg}$ en el periodo de julio a diciembre de 2004 y $50 \mathrm{mg} / \mathrm{kg}$ a partir de enero de 2005. La determinación del contenido de azufre en las muestras recogidas se realizó empleando el procedimiento normalizado adaptado a este tipo de producto. La totalidad de las gasolinas analizadas presentaron un contenido de azufre inferior al límite máximo establecido por la legislación. Estos resultados muestran la rápida adaptación llevada a cabo por las refinerías en sus procesos de desulfurización para cumplir con la nueva legislación.

Palabras clave: azufre, desulfurización, gasolina, legislación, refinería

\section{Evolution of Sulphur Content in Commercialized Gasoline in Galicia (Spain) as a Result of the Application of the Royal Decree 1700/2003}

\begin{abstract}
The objective of the study was to determine the sulphur content in gasoline which were commercialized in Galicia six months before and six months after the first of January 2005, the date established by law, according to the royal decree $1700 / 2003$, to reduce the sulphur content in gasoline. This was done to verify the fulfilment of the new legislation starting that date: $150 \mathrm{mg} / \mathrm{kg}$ in the period July to December 2004 and $50 \mathrm{mg} / \mathrm{kg}$ in the period January to June 2005. The sulphur content in the samples was determined according to a normalized procedure adapted to this type of product. All gasoline samples presented sulphur content lower than the maximum limit established by the legislation. These results show the fast adaptation performed by the refineries in their desulphurization processes to comply with the new legislation.
\end{abstract}

Keywords: sulphur, desulphurization, gasoline, legislation, refinery 


\section{INTRODUCCIÓN}

La polución del aire, principalmente en áreas urbanas, es uno de los mayores problemas de este siglo (Landulfo et al., 2007). En los países desarrollados, donde más del $75 \%$ de la población vive en áreas urbanas, los recursos económicos de los que disponen, permiten la adopción de medidas efectivas contra la polución y el impacto generados por los contaminantes (Özden et al., 2008) situación que por el contrario no se repite en los países en vías de desarrollo. En los últimos años, la prevención y el control de la polución han ganado importancia (Bailey y Solomon, 2004) aunque no por ello se ha resuelto el problema ya que todavía existen muchas ciudades en el mundo expuestas a altos niveles de polución (Castabile y Allegrini, 2008). Son muchas las razones para explicar este cambio en la calidad del aire, entre ellas y fundamentalmente, que la rápida urbanización e industrialización sufrida por muchos países ha provocado un incremento de las emisiones contaminantes, debidas al transporte, producción de energía y actividad industrial, actividades todas ellas concentradas en áreas urbanas. Los impactos medioambientales llegan a ser importantes en ciudades de China e India, donde se combina la fuerte expansión industrial, la alta densidad de población y el elevado número de vehículos (Gurjar et al., 2008). En Europa, el transporte es el máximo responsable de las emisiones contaminantes en las ciudades (Lumbreras et al., 2008).

En los últimos 30 años se ha experimentado una gran variación de los requisitos de calidad de los combustibles y carburantes, no son sólo el resultado de consideraciones acerca de la evolución y diseño de los motores y tecnologías desarrolladas, sino principalmente son consecuencia de la creciente preocupación por la protección del medio ambiente. Uno de los parámetros destinados a controlar la calidad de los combustibles es el contenido de azufre, parámetro de suma importancia debido a los problemas que origina tanto al propio equipo, como al medio ambiente y a la salud. El azufre presente en el crudo y por lo tanto en los productos de él derivados, es por sí mismo un contaminante, pero aún más importante es que el azufre impide la adopción de las principales tecnologías para el control de la contaminación resultando únicamente efectivas cuando el contenido de azufre es cercano a cero (Blumberg et al., 2003)

Son muchos los problemas asociados a la presencia de compuestos de azufre en las gasolinas:

a) los compuestos de azufre $\left(\mathrm{SO}_{\mathrm{x}}\right)$ emitidos en los gases de exhaustación de los vehículos son los principales responsables de la polución del aire (Cofala et al., 2004).

b) los vehículos de encendido por chispa, en los que la gasolina es utilizada como combustible, están provistos actualmente de convertidores catalíticos diseñados para reducir las emisiones de monóxido de carbono, hidrocarburos inquemados y óxidos de nitrógeno en un $90 \%$. La utilización de gasolina con alto contenido de azufre, hace disminuir la eficiencia de estos catalizadores llamados catalizadores de 3 vías (de Lasa et al., 2006). La reducción de la actividad catalítica se debe a que las especies de azufre son adsorbidas en los centros activos del catalizador evitando que se lleven a cabo las reacciones catalíticas de interés e interfieren en la disponibilidad del oxígeno en la superficie del catalizador, cuya concentración es fundamental para maximizar la reducción de las emisiones de $\mathrm{NO}_{x}$. La utilización de gasolinas con alto contenido de azufre provoca efectos a corto plazo debido a la adsorción de los compuestos de azufre en la superficie del catalizador, efectos a medio plazo debido a la penetración del azufre en el metal precioso del catalizador y en el material que se ocupa del almacenamiento del oxígeno y efectos a largo plazo debido a la irreversibilidad de dicho proceso (Schifter et al., 2003).

c) los compuestos de azufre favorecen la corrosión del equipamiento (Siddiqui et al., 2006).

En los últimos años, las regulaciones impuestas a los combustibles han estado orientadas principalmente a limitar el contenido de azufre, el cual se ha visto drásticamente minimizado a fin de combatir la polución del aire. En EEUU y Canadá el contenido de azufre máximo permitido en gasolina fue fijado en 30 ppm para el año 2005.

La Unión Europea también ha tomado medidas al respecto a través de la Directiva 98/70/CE (1998) que establece, por motivos relacionados con la salud y el medio ambiente, especificaciones mínimas 
para los combustibles destinados a ser utilizados en vehículos equipados con motores de explosión o de compresión. La Directiva 98/70/CE (1998), traspuesta a la legislación nacional de cada país miembro de la Unión Europea y de obligado cumplimiento establece los parámetros destinados a asegurar la calidad de los combustibles así como sus valores límite. Respecto al contenido de azufre se fijó como valor máximo permitido $500 \mathrm{mg} / \mathrm{kg}$, valor modificado con la entrada en vigor de la Directiva 2003/17/CE (2003), que deja fijado el contenido de azufre en $150 \mathrm{mg} / \mathrm{kg}$ a partir del año 2000.

En España el Real Decreto 1700/2003 (R.D., 2003), ha transpuesto la Directiva Europea 2003/17/CE (2003). En el artículo 1 de este Real Decreto referente a las especificaciones de gasolinas, se establece que el contenido máximo de azufre en gasolinas no deberá superar el valor de $50 \mathrm{mg} / \mathrm{kg}$ a partir del 1 de enero de 2005, lo que supone una reducción de $100 \mathrm{mg} / \mathrm{kg}$ respecto al límite máximo establecido anteriormente.

Los límites máximos permitidos por la legislación española para el contenido de azufre en la gasolina comercializada en España desde el año 1989 hasta el 2009 se muestran en la figura 1.

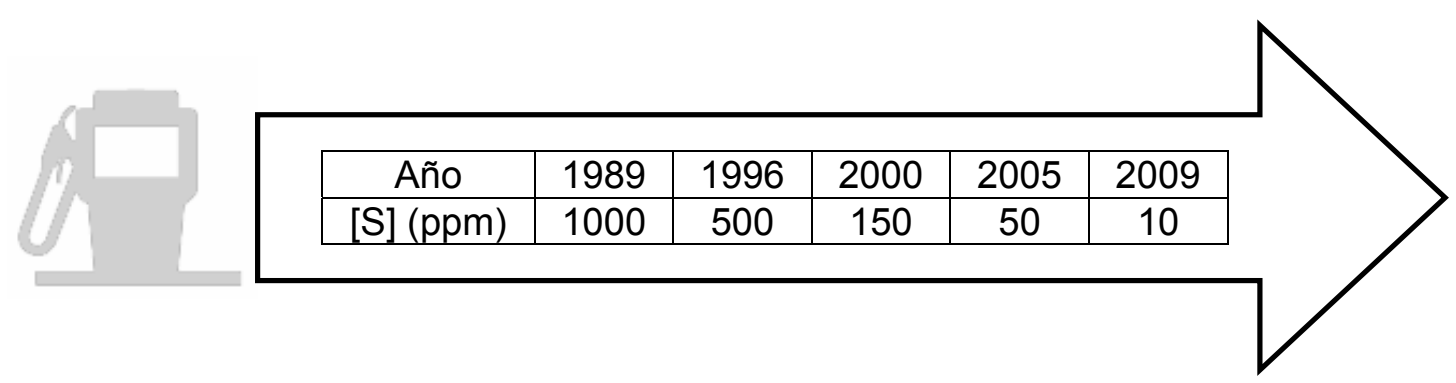

Fig. 1. Evolución del contenido de azufre (ppm) en la gasolina.

Las exigencias de la legislación vigente en cuanto a la reducción del contenido de azufre en los combustibles, han tenido consecuencias para las refinerías, donde la eficiencia de los procesos de desulfuración aplicados tanto a gasolinas como a gasóleos y kerosenos, han llegado a ser el punto clave. La gasolina comercial actual está formada por blending, es decir, por combinación de diferentes corrientes de refinería: destilación directa, unidad de craqueo catalítico (FCC) y unidad de coquización. La gasolina obtenida de la unidad de FCC contribuye con más del $90 \%$ al contenido de azufre presente en la gasolina comercial (Yin y Xia, 2004) por lo que la desulfuración de esta corriente resulta fundamental a la hora de cumplir con los requerimientos establecidos por la legislación. El contenido de azufre en las gasolinas comerciales obtenidas por el resto de las corrientes de refinería no resulta un problema a la hora de cumplir con la legislación vigente aunque sí para producir gasolina con un contenido de azufre inferior a $30 \mathrm{mg} / \mathrm{kg}$ haciéndose necesaria igualmente la desulfuración (Babich y Moulijn, 2003).

Los procesos de hidrodesulfuración convencional (HDS) que venían siendo aplicados en las refinerías no resultan efectivos a la hora de reducir el contenido de azufre a niveles próximos a cero y además provocan la hidrogenación de las olefinas con la consecuente pérdida en el número de octano por lo que muchas investigaciones han estado orientadas al estudio de métodos alternativos de desulfuración así como a la mejora del proceso de hidrodesulfuración (Ali et al., 2006; Ito y Rob, 2006; Wang et al., 2007).

El objetivo de este estudio es comprobar si las gasolinas comercializadas en Galicia (España) cumplen con lo establecido en la legislación antes y después de la entrada en vigor del Real Decreto 1700/2003 (2003), llevando a cabo para ello la determinación del contenido de azufre según lo establecido por la norma ASTM D-1552-03 (2006) adaptada a este tipo de producto. Los resultados obtenidos demostraron que todas las gasolinas analizadas presentan un contenido en azufre inferior al límite máximo establecido por la legislación en cada uno de los semestres en los que ha sido llevado a cabo el estudio y en los que el contenido máximo de azufre permitido fue reducido de 150 $\mathrm{mg} / \mathrm{kg}$ a $50 \mathrm{mg} / \mathrm{kg}$. 


\section{PROCEDIMIENTO}

\section{Recolección y almacenamiento de muestras}

Las muestras de gasolina fueron tomadas en estaciones de servicio ubicadas en las cuatro provincias gallegas (A Coruña, Lugo, Ourense y Pontevedra). Se ha realizado previamente un estudio del número y distribución de los posibles puntos de toma de muestra en cada provincia con el fin de garantizar que dicha selección fuese lo más representativa posible de la totalidad de las gasolinas comercializadas en toda el área de estudio seleccionada. Las provincias costeras de Pontevedra y A Coruña poseen una mayor densidad de población y como consecuencia un mayor número de puntos de distribución de gasolina, por lo que en ellas y para garantizar la representatividad del estudio, fueron recogidas un mayor número de muestras que en las provincias interiores de Lugo y Ourense. El periodo de muestreo y la frecuencia de toma de muestras así como el número total de muestras fueron establecidos previamente con el fin de conseguir que de los resultados obtenidos de dicho estudio se pudiesen derivar conclusiones fidedignas acerca del estado actual de la calidad de las gasolinas comercializadas en Galicia.

Se estableció una frecuencia de recogida de muestras mensual y durante un periodo de un año, comprendido entre Julio de 2004 hasta Junio de 2005 intervalo de tiempo que corresponde a los seis meses anteriores y posteriores a la entrada en vigor del Real Decreto 1700/2003 (2003). El número óptimo de muestras a recoger quedó establecido entre 300 y 400 para así poder disponer de un número de muestras significativo de cada uno de los tipos de gasolina presentes en el mercado, en ese momento, de acuerdo a su número de octano (10 95, 1098 e 10 97). Se recogieron un total de 383 muestras de gasolina de diferente octanaje en todo el territorio gallego cuya distribución geográfica se muestra en la Figura 2.

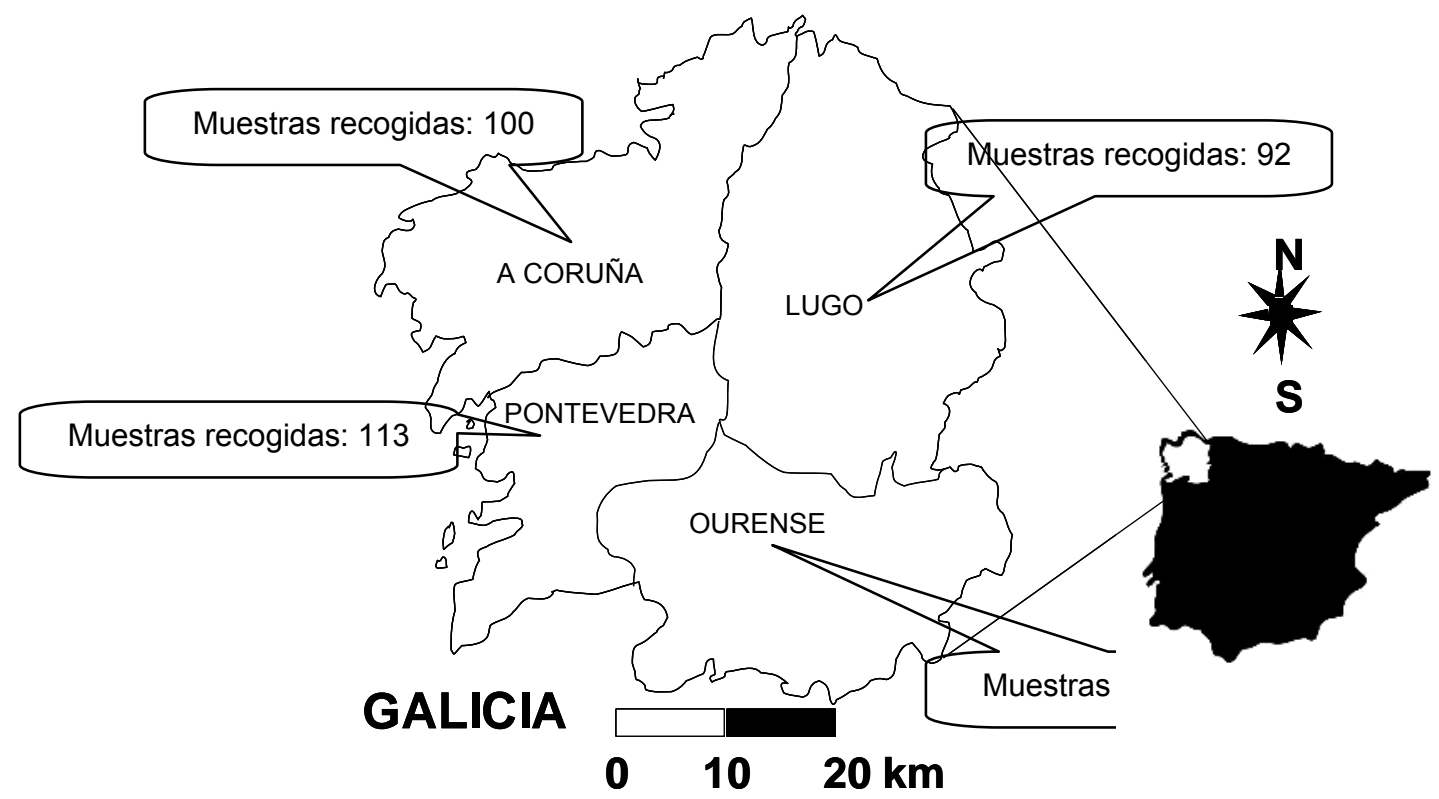

Fig.2: Distribución geográfica de las muestras de gasolina recogidas durante el periodo de estudio.

Las muestras fueron recogidas siguiendo rigurosamente el procedimiento descrito en la norma UNE-EN 14275 (2004), norma establecida para llevar a cabo la toma de muestra de combustibles de automoción en bombas y surtidores comerciales. Para la recogida de las muestras de gasolina se utilizaron botellas de $2 \mathrm{~L}$ de capacidad provistas de arandela y tapa roscada para conservar la integridad de la muestra y el llenado de las mismas fue realizado adoptando las precauciones de seguridad que deben cumplirse tal y como se recoge en la ficha de datos de seguridad del producto y en la norma UNE-EN ISO 3170 (1999), indicada para la toma de muestras manual de productos petrolíferos líquidos. Las muestras fueron correctamente etiquetadas y selladas y posteriormente conservadas a $4^{\circ} \mathrm{C}$ hasta su análisis con el fin de evitar posibles pérdidas de los elementos más volátiles. 
La clasificación de las muestras de gasolina recogidas según su octanaje durante el periodo de estudio correspondiente al segundo semestre del año 2004 y primer semestre del año 2005, se recogen en la Tabla 1.

Tabla 1: Muestras de gasolina recogidas en función de su índice de octano.

\begin{tabular}{|l|c|c|c|c|}
\hline \multirow{2}{*}{$N^{\circ}$ MUESTRAS } & \multicolumn{3}{|c|}{ INDICE DE OCTANO } & \multirow{2}{*}{ TOTAL } \\
\cline { 2 - 4 } & IO 95 & IO 98 & IO 97 & \\
\hline $2^{\circ}$ semestre 2004 & 73 & 47 & 68 & 188 \\
\hline $1^{\text {er }}$ semestre 2005 & 76 & 54 & 65 & 195 \\
\hline
\end{tabular}

\section{Método}

La concentración de azufre en las muestras de gasolina se determinó siguiendo el procedimiento descrito en la norma ASTM D-1552-03 (2006), adaptando dicho procedimiento a este tipo de producto. El método se basa en la combustión de la muestra y posterior detección del $\mathrm{SO}_{2}$ producido mediante detección infrarroja. La muestra es colocada en un horno de combustión en una atmósfera rica en oxígeno y a una temperatura de $1350^{\circ} \mathrm{C}$. Las condiciones de temperatura y el flujo constante de oxígeno en el horno causan la combustión de la muestra: el azufre contenido en ella es liberado y oxidado posteriormente a $\mathrm{SO}_{2}$. Los gases producidos son conducidos hasta la parte final del tubo de combustión, zona de mayor temperatura, donde permanecerán el tiempo necesario para permitir que la oxidación del azufre sea llevada a cabo de forma eficiente. El flujo de gases producido de la combustión de la muestra, pasa a través de dos tubos de cloruro cálcico que eliminan la humedad de la muestra, a través de un regulador de flujo que fija el flujo de gases de la muestra y, posteriormente a través de la celda de detección infrarroja que mide la concentración de $\mathrm{SO}_{2}$ generada. Un microprocesador calcula la concentración de azufre presente en la muestra a partir del peso inicial de la misma, la señal integrada del detector y la recta de calibración obtenida anteriormente al análisis de las muestras.

La concentración de azufre en las muestras de gasolina, fue determinada utilizando el analizador elemental LECO SC-144DR (analizador de azufre y carbono) cuya sensibilidad permite la cuantificación de azufre en el rango de concentración de $5 \mathrm{mg} / \mathrm{kg}$ a $26 \%(\mathrm{~m} / \mathrm{m})$ y con una precisión menor o igual al $1 \%$ de la desviación estándar relativa.

El software del equipo ofrece la posibilidad de crear métodos específicos dependiendo de las propiedades de la muestra que se desea analizar. Para cada tipo de muestra, es necesario fijar el tiempo mínimo y máximo de integración, parámetro de gran importancia en este análisis debido a la diferente velocidad de combustión de las muestras.

Para la determinación de la concentración de azufre en gasolinas se fijó un tiempo mínimo de integración de 30 segundos y un tiempo máximo de 90 segundos. Se determinó el valor de la señal del blanco de análisis para conocer la señal residual del equipo y poder corregir las variaciones de concentración producidas ajenas a la propia muestra.

La calibración del equipo fue llevada a cabo utilizando patrones de calibración certificados de diferente contenido en azufre ( $1 \mathrm{mg} / \mathrm{kg}, 10 \mathrm{mg} / \mathrm{kg}, 35 \mathrm{mg} / \mathrm{kg}, 100 \mathrm{mg} / \mathrm{kg}$ y $1000 \mathrm{mg} / \mathrm{kg}$ ) y en matriz gasolina. Cada uno de los patrones fue analizado 3 veces, tomando pesos diferentes cada vez para asegurar una correcta calibración del equipo. Cuando el equipo finaliza el análisis de la muestra conocida, la curva de calibración compara el área medida del azufre con el área correcta de azufre creada, calculada al multiplicar el peso de la muestra por el porcentaje de azufre del patrón. La curva de calibración es utilizada después para calcular el área correcta de azufre de las muestras desconocidas.

Se pesaron alrededor de 0,1 gramos de muestra de gasolina en una navecilla de cerámica donde en un tercio de su capacidad se puso previamente un compuesto acelerante de la combustión (en este 
caso el producto comercial Com-Aid) y se distribuyó la muestra por toda la superficie de la navecilla con el propósito de favorecer la combustión homogénea de la muestra y obtener así resultados más consistentes. Después de pesada la muestra, se añade de nuevo Com-Aid hasta llenar dos tercios de la capacidad de la navecilla y posteriormente se introduce la navecilla en el horno de combustión mantenido a una temperatura de $1350^{\circ} \mathrm{C}$ y con un flujo constante de oxígeno de $3,5 \mathrm{ml} / \mathrm{min}$. El detector registra la señal de azufre presente en la muestra y el software del equipo compara el área obtenida para la muestra y la anteriormente obtenida para los patrones, obteniéndose así la concentración de azufre presente en las muestras. Cada una de las muestras fue analizada 3 veces.

\section{RESULTADOS Y DISCUSIÓN}

Se llevó a cabo la determinación del contenido de azufre en las 383 muestras de gasolina de diferente número de octano (95, 98 y 97 ) recogidas en Galicia. El contenido de azufre obtenido para cada una de ellas corresponde al valor medio calculado a partir de tres determinaciones y calculando la desviación de los datos de acuerdo con las características de precisión del equipo.

Muestras de gasolina recogidas en el $2^{\circ}$ semestre del 2004

En este periodo se recogieron un total de 188 muestras de gasolina de diferente octanaje: 72 de 10 95, 47 de 1098 y 68 de 10 97, cuya distribución es acorde al consumo real de gasolinas en Galicia.

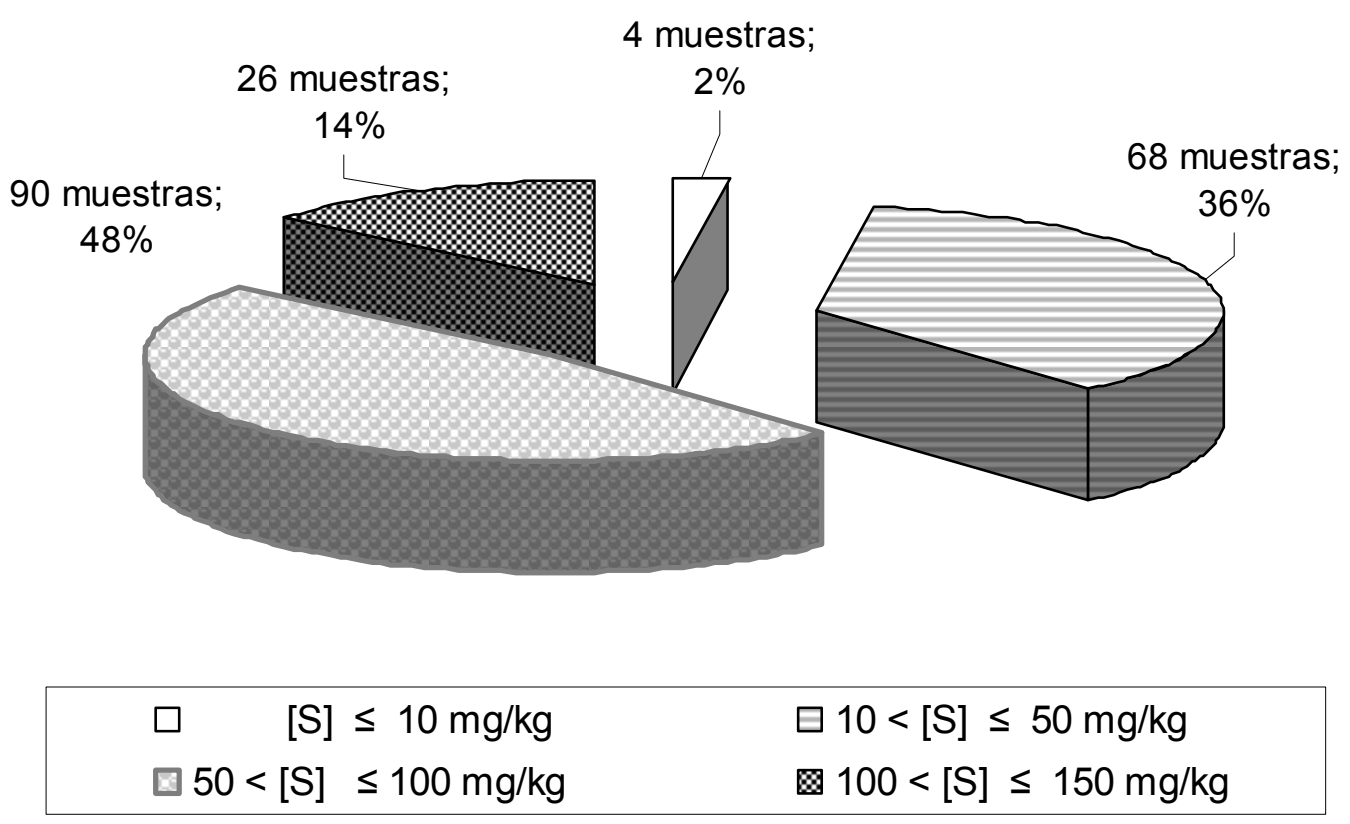

Fig. 3: Porcentaje muestras gasolina- Intervalo de concentración azufre ([S] en mg/kg).

El contenido de azufre obtenido para todas las muestras de gasolina recogidas en el segundo semestre del año 2004, se encuentra por debajo de $150 \mathrm{mg} / \mathrm{kg}$, límite máximo establecido por la legislación en este semestre y antes de la entrada en vigor del Real Decreto 1700/2003.

Se estudiaron los resultados obtenidos en función del intervalo de concentración en el que se encuentran. Se clasificó el contenido de azufre en cuatro diferentes intervalos de concentración: contenido menor o igual de $10 \mathrm{mg} / \mathrm{kg}$, mayor de 10 y menor o igual de $50 \mathrm{mg} / \mathrm{kg}$, mayor de 50 y menor o igual de $100 \mathrm{mg} / \mathrm{kg}$ y mayor de 100 y menor o igual de $150 \mathrm{mg} / \mathrm{kg}$, encontrándose que el $62 \%$ de las muestras presentan un contenido de azufre en el intervalo de 150 a $50 \mathrm{mg} / \mathrm{kg}$ y un $38 \%$ de las muestras presentan un contenido de azufre menor de 50 mg/kg (Figura 3).

La comparación entre los diferentes tipos de gasolina y el contenido de azufre encontrado en ellas, demostró que la gasolina de 1098 es la que presenta un menor contenido de azufre y mayor porcentaje de muestras con un contenido de azufre menor de $10 \mathrm{mg} / \mathrm{kg}$ (Figura 4). 


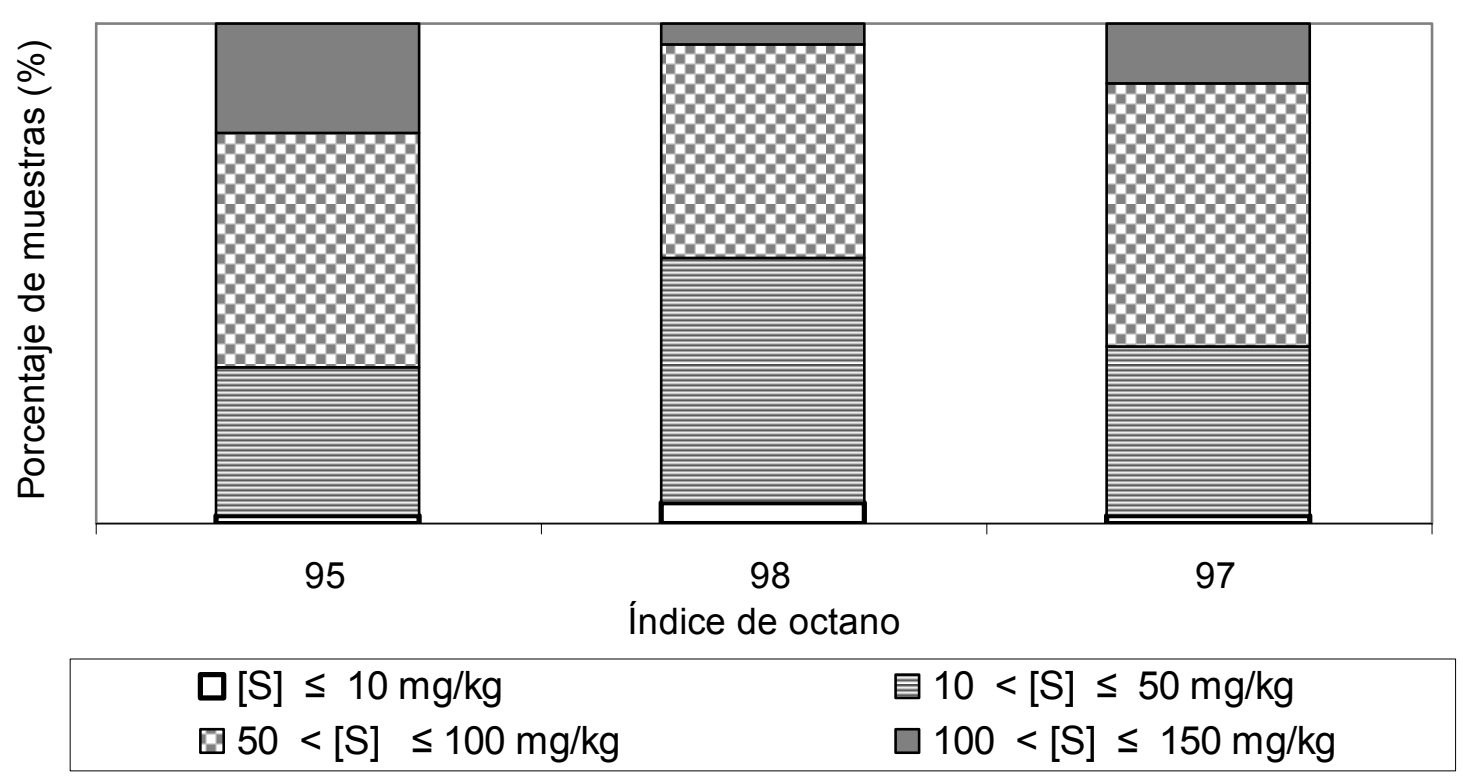

Fig. 4: Porcentaje muestras gasolina según IO - Intervalo de concentración azufre ([S] en mg/kg).

Muestras de gasolina recogidas en el $1^{\text {er }}$ semestre del 2005

En este periodo se recogieron un total de 195 muestras de gasolina de diferente octanaje: 76 de 10 95, 54 de 1098 y 65 de IO 97, cuya distribución es acorde con el consumo real de Galicia.

Los resultados obtenidos en la determinación del contenido de azufre, demuestran que todas las muestras analizadas presentan un contenido de azufre inferior a $50 \mathrm{mg} / \mathrm{kg}$, límite máximo permitido por la legislación para este semestre de acuerdo a la entrada en vigor del Real Decreto 1700/2003.

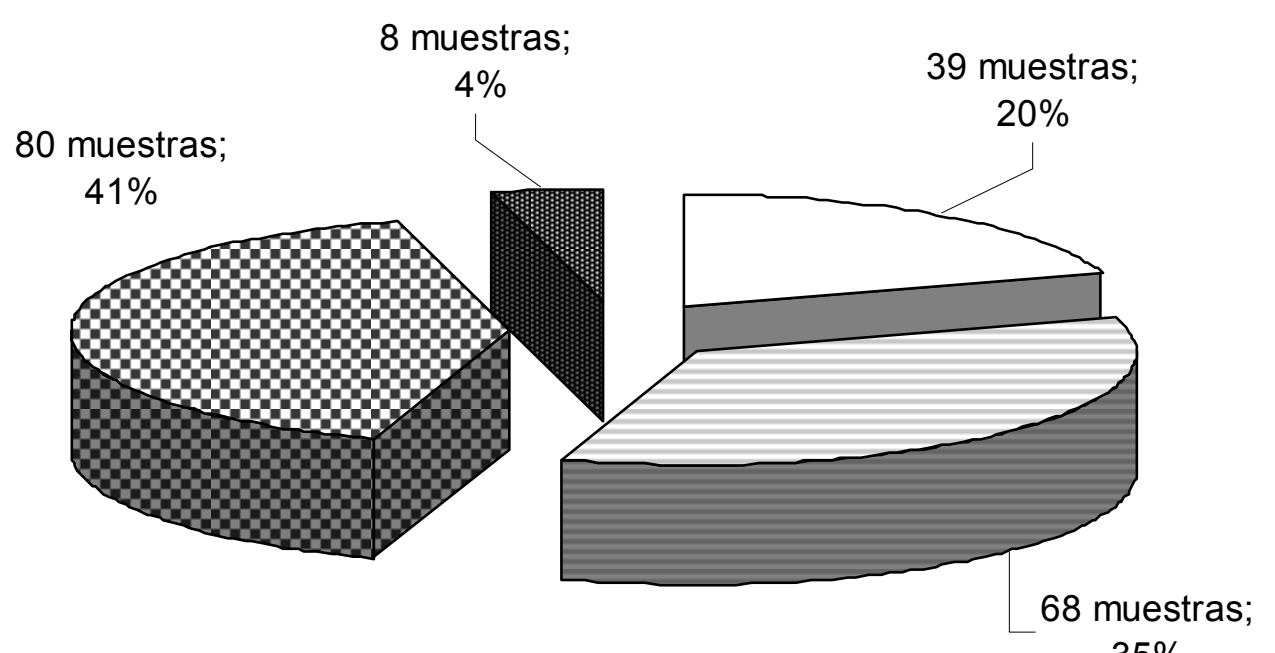

$35 \%$

$$
\begin{array}{ll}
\square \quad[S] \leq 10 \mathrm{mg} / \mathrm{kg} & \boxminus 10<[\mathrm{S}] \leq 20 \mathrm{mg} / \mathrm{kg} \\
\square 20<[\mathrm{S}] \leq 40 \mathrm{mg} / \mathrm{kg} & \text { 薢 } 40<[S] \leq 50 \mathrm{mg} / \mathrm{kg}
\end{array}
$$

Fig.5: Porcentaje de muestras gasolinas- Intervalo de contenido de azufre ([S] en mg/kg). 
Se estudiaron los resultados obtenidos en función del intervalo de concentración en el que se encuentran. Se clasificó el contenido de azufre en cuatro diferentes intervalos de concentración: contenido menor o igual de $10 \mathrm{mg} / \mathrm{kg}$, mayor de 10 y menor o igual de $20 \mathrm{mg} / \mathrm{kg}$, mayor de 20 y menor o igual de $40 \mathrm{mg} / \mathrm{kg}$ y mayor de 40 y menor o igual de $50 \mathrm{mg} / \mathrm{kg}$, encontrándose que el $80 \%$ de las muestras presentan un contenido de azufre en el intervalo de 50 a $10 \mathrm{mg} / \mathrm{kg}$ y un $20 \%$ de las muestras presentan un contenido de azufre menor de $10 \mathrm{mg} / \mathrm{kg}$ (Figura 5).

La comparación entre los diferentes tipos de gasolina y el contenido de azufre encontrado en ellas, demostró que la gasolina de 1098 es la que presenta un menor contenido de azufre no presentando ninguna de las muestras analizadas un contenido superior a $40 \mathrm{mg} / \mathrm{kg}$ y un mayor porcentaje de muestras con un contenido de azufre menor de $10 \mathrm{mg} / \mathrm{kg}$ (Figura 6).

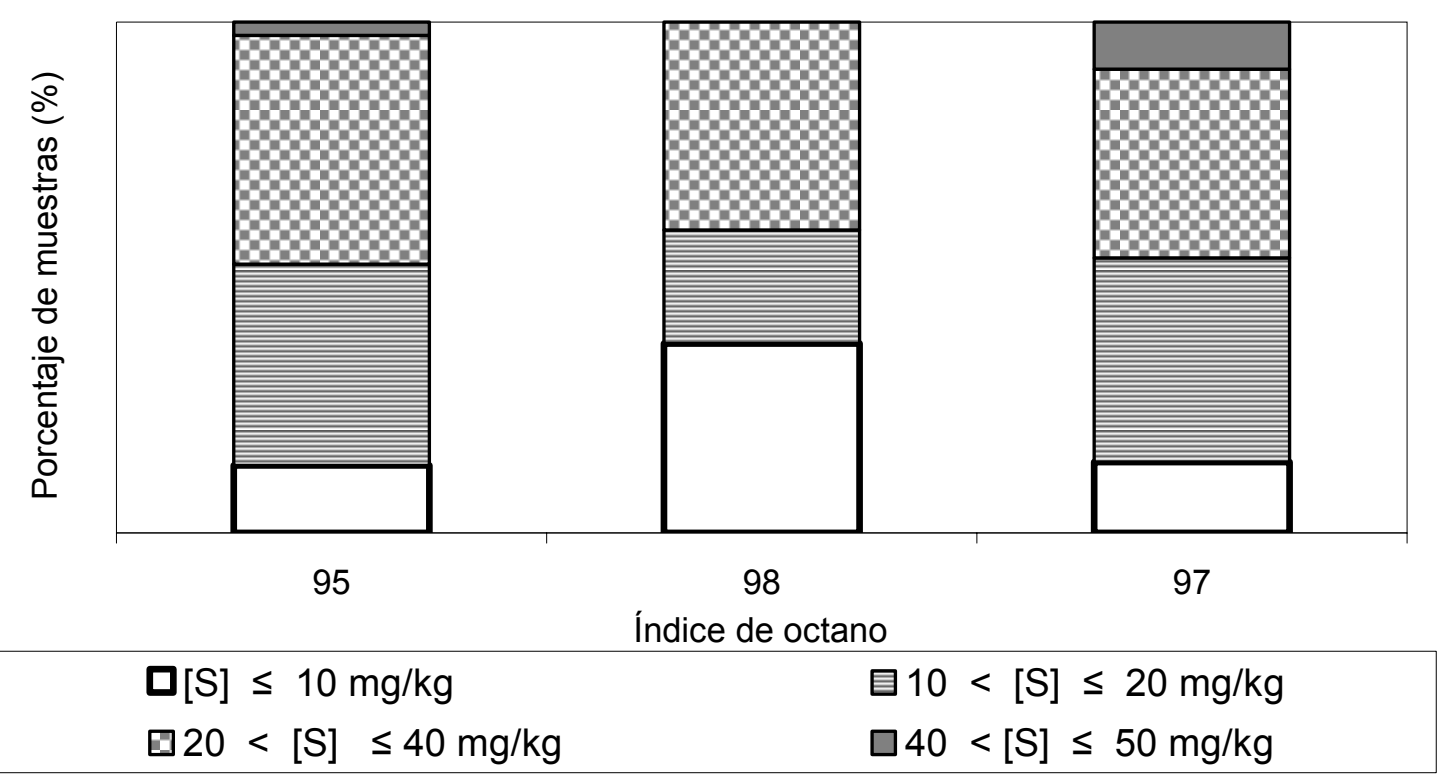

Fig. 6: Porcentaje muestras gasolina según I.O- Intervalo de concentración azufre ([S] en mg/kg).

\section{Evolución del contenido de azufre en las muestras de gasolina analizadas.}

Todas las gasolinas analizadas, independientemente de su número de octano, presentan un contenido de azufre menor que el límite máximo establecido por la legislación española en el periodo de tiempo que abarca la realización de este estudio: de Julio a Diciembre del 2004 cuyo valor máximo permitido era $150 \mathrm{mg} / \mathrm{kg}$ y de Enero a Julio del 2005 donde el valor máximo permitido para el contenido de azufre era de $50 \mathrm{mg} / \mathrm{kg}$. La evolución en el contenido de azufre presente en las gasolinas de IO 95, IO 98 e IO 97 analizadas se muestra en las Figuras 7, 8 y 9.

La drástica disminución observada en el contenido de azufre en dichas figuras se corresponde con la fecha (enero-2005) de entrada en vigor del nuevo valor máximo permitido de $50 \mathrm{mg} / \mathrm{kg}$. De este modo se constata la rápida optimización y adaptación de los procesos de refinería para cumplir con la legislación vigente.

Realizando una comparativa de la evolución de los tres tipos de gasolina se observa que el contenido de azufre presente en la gasolina de 98 octanos alcanza valores menores que en las gasolinas de 95 y 97 octanos a partir de enero de 2005, apareciendo ya muestras de gasolina de 98 octanos con un contenido de azufre inferior a $10 \mathrm{mg} / \mathrm{kg}$, límite que está previsto que entre en vigor en el año 2009 (R.D. 1700/2003). Los resultados obtenidos demuestran la rápida evolución de la presencia en el mercado gallego de gasolinas de cada vez menor contenido en azufre con una significativa y relevante presencia cada vez mayor de aquellas que tienen menos de $10 \mathrm{mg} / \mathrm{kg}$ de azufre, lo que redunda en una mayor y mejor protección del medio ambiente gracias a la menor contaminación por problemas derivados de las emisiones producto de la combustión originada por este tipo de combustible/carburante. 


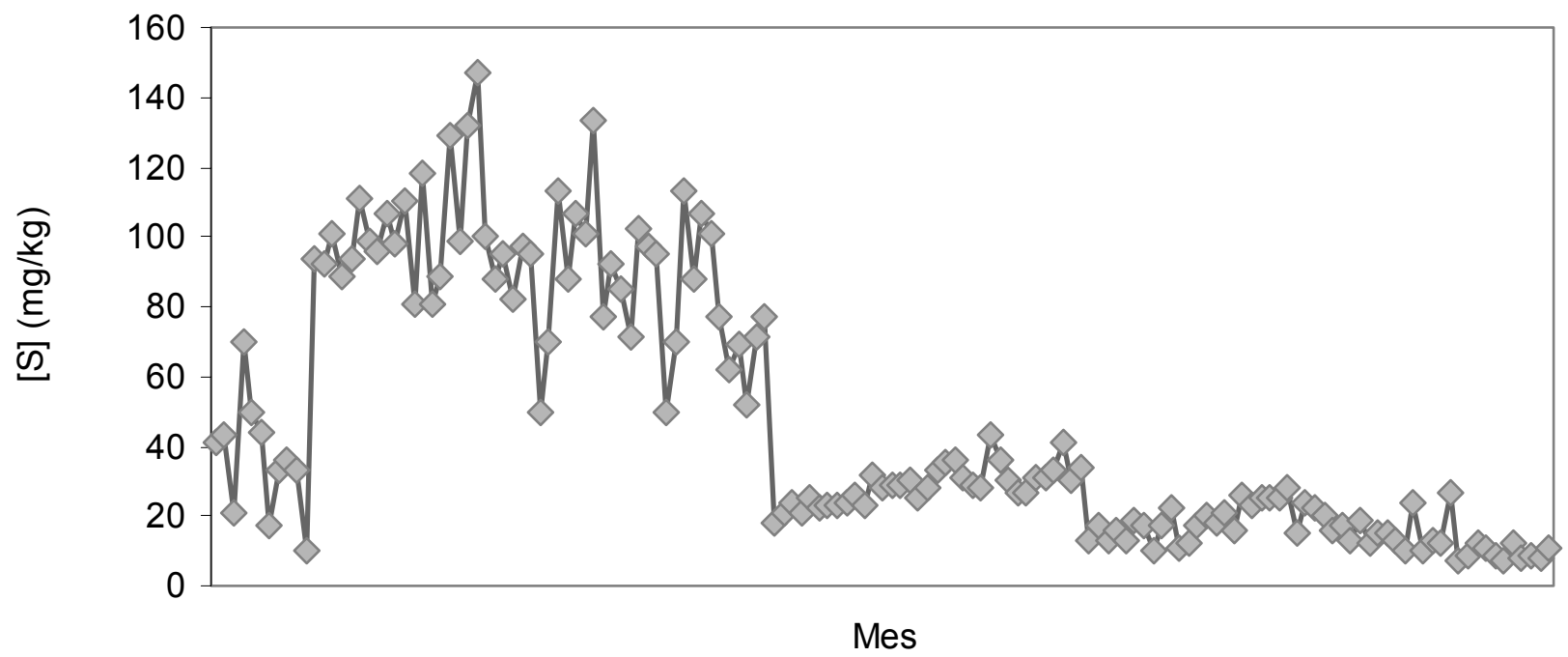

Fig. 7. Contenido de azufre $(\mathrm{mg} / \mathrm{kg})$ en las muestras de gasolina de $\mathrm{IO} 95$ durante el periodo de estudio (julio 2004 - junio 2005).

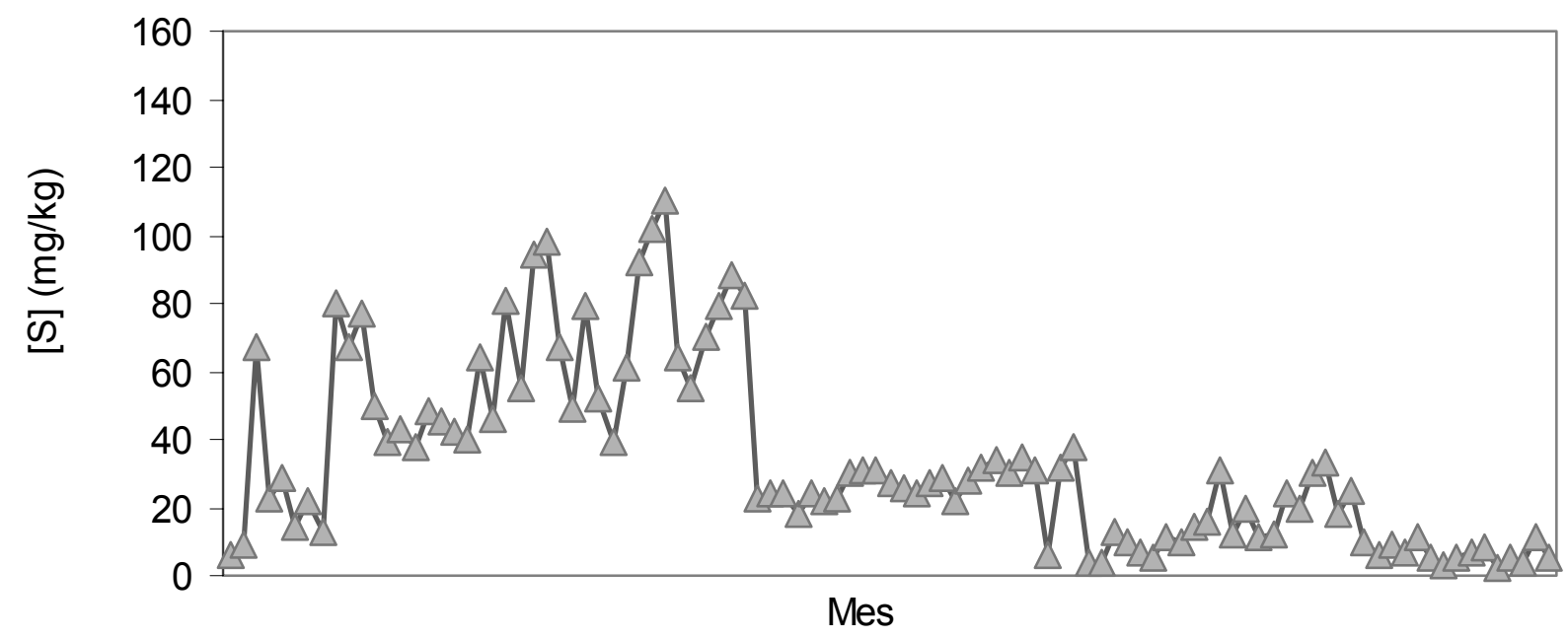

Fig.8. Contenido de azufre $(\mathrm{mg} / \mathrm{kg})$ en las muestras de gasolina de 1.0 .98 durante el periodo de estudio (julio 2004 - junio 2005).

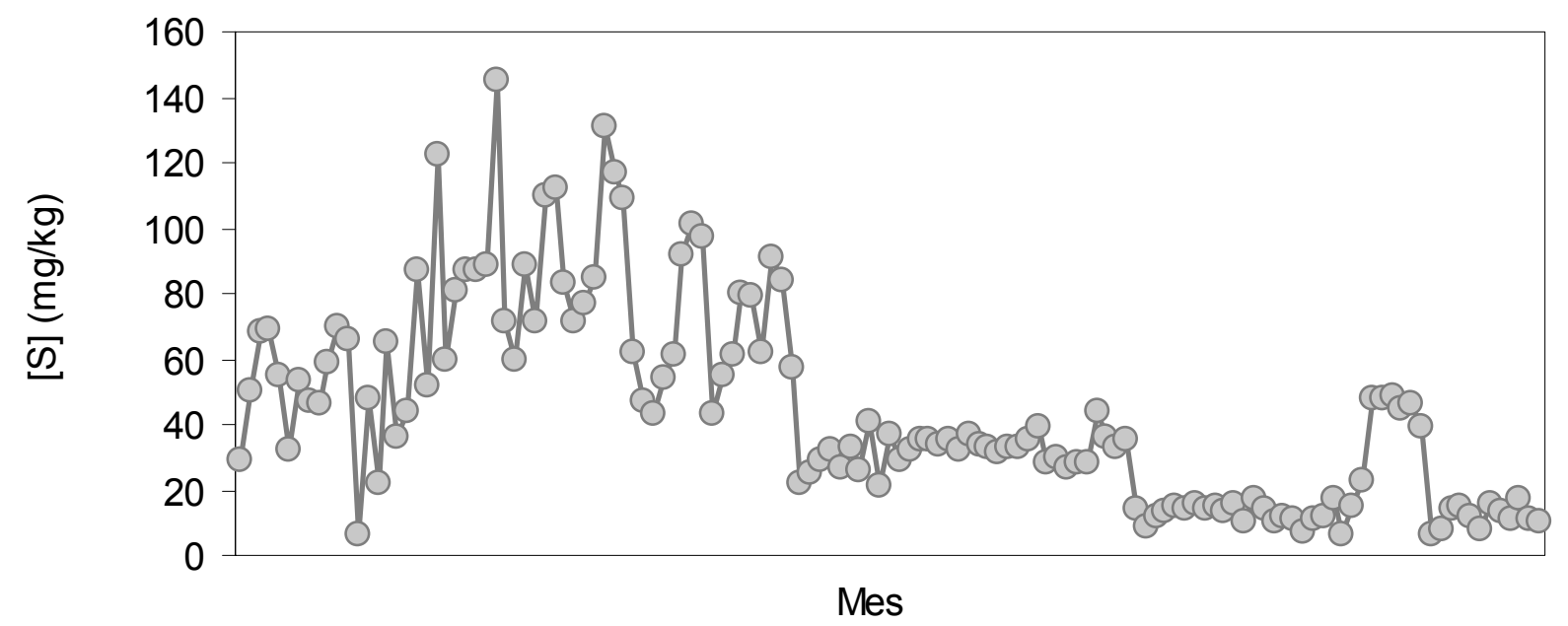

Fig. 9. Contenido de azufre $(\mathrm{mg} / \mathrm{kg})$ en las muestras de gasolina de 1097 durante el periodo de estudio (julio 2004 - junio 2005). 
Estudio comparativo entre las muestras recogidas durante el primer y segundo semestre

El contenido de azufre presente en las gasolinas recogidas fue analizado realizando una comparación entre las muestras recogidas en el segundo semestre del 2004 y en el primer semestre del 2005 con el objetivo de comprobar si existe diferencia estadística significativa entre ambas como parece desprenderse de la observación de las figuras anteriores, así como la existencia de valores extremos y/o atípicos.

El análisis estadístico comparativo entre ambas poblaciones muestrales se realizó mediante la construcción de diagramas de cajas y bigotes y a partir de ellos se estudió la distribución de los datos de la población. Las figuras 10, 11 y 12 muestran la distribución por semestres de cada uno de los tipos de gasolina recogidos.

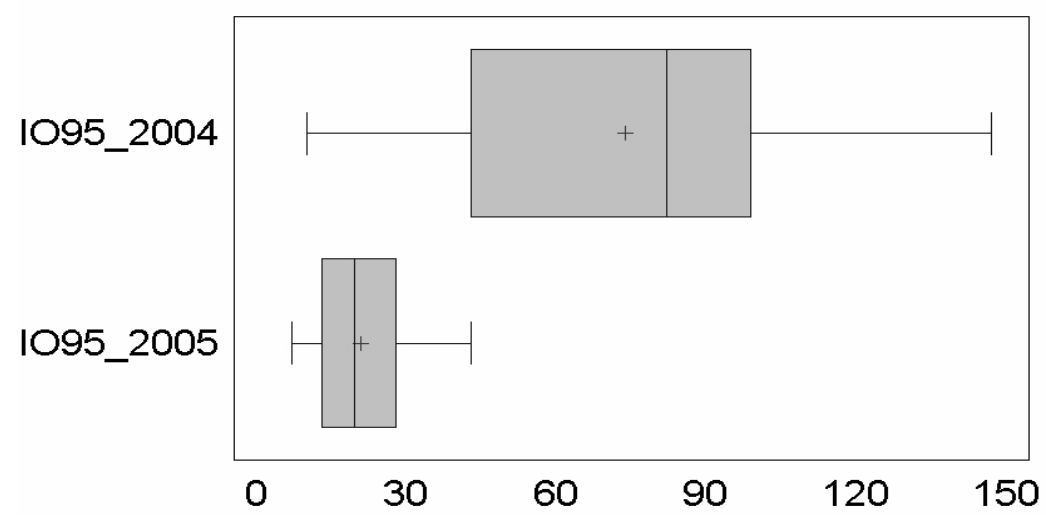

Fig. 10: Distribución de los datos de ambas poblaciones para la gasolina de 95 octanos.

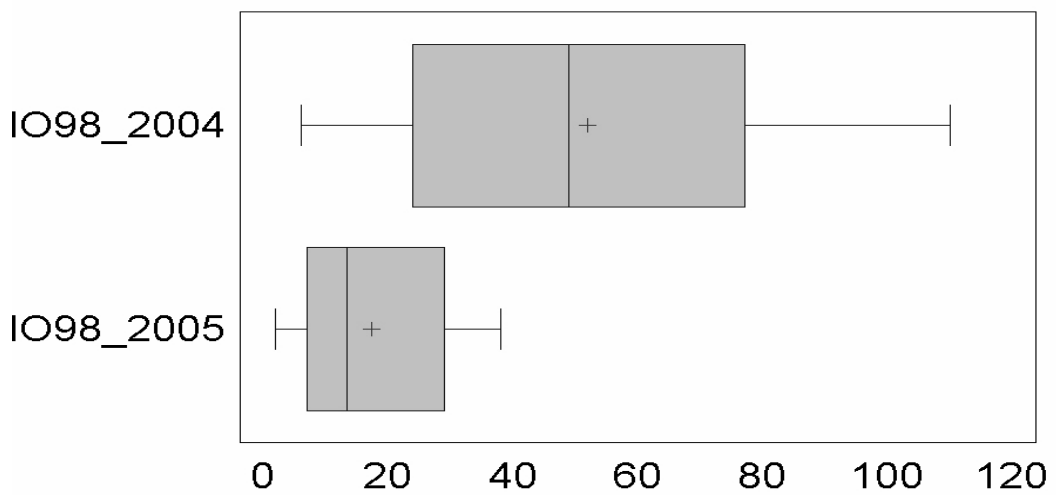

Fig. 11: Distribución de los datos de ambas poblaciones para la gasolina de 98 octanos

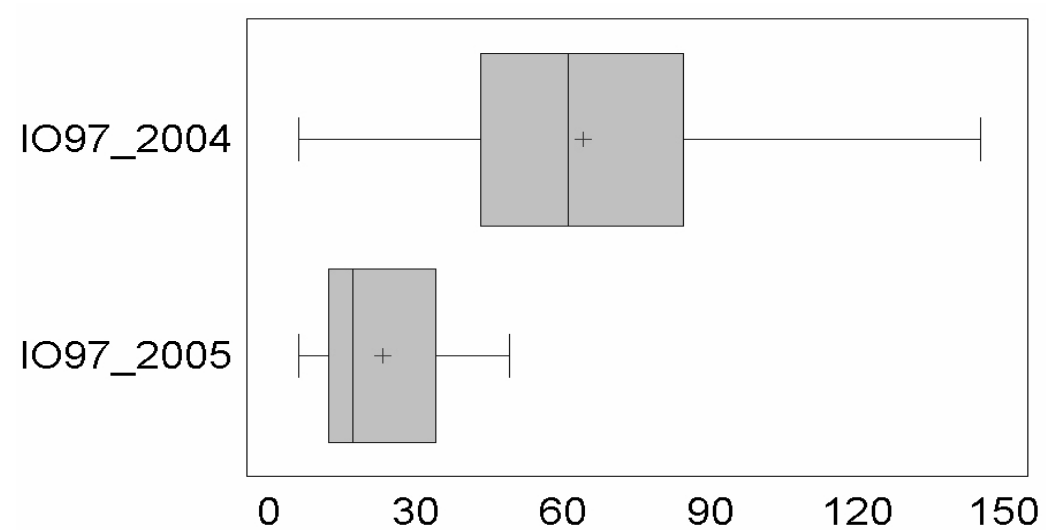

Fig.12: Distribución de los datos de ambas poblaciones para la gasolina de 97 octanos. 
En cada una de las figuras se muestran dos gráficos de cajas y bigotes correspondientes a las muestras tomadas en el año 2004 y 2005 respectivamente. El comportamiento observado por comparación de ambas poblaciones muestrales resulta similar para los diferentes tipos de gasolinas, y se puede decir que:

- las gasolinas de 95, 98 y 97 octanos tomadas en el periodo correspondiente al año 2004 presentan una variabilidad no homogénea. Lo mismo sucede en el caso de las gasolinas de 95, 98 y 97 octanos tomadas en el periodo correspondiente al año 2005.

- la dispersión de los datos de las gasolinas tomadas en el año 2004 es mayor que la dispersión observada para las gasolinas tomadas en el año 2005, la dispersión de los datos está relacionada con la anchura del lado mayor de la caja.

- la longitud de los bigotes indica el intervalo de valores en el que se trabaja, por lo tanto la dispersión de la serie de datos. De nuevo se puede observar que la dispersión de los datos es mayor para las gasolinas recogidas durante el año 2004 que para las recogidas en el 2005.

\section{CONCLUSIONES}

De los resultados de este estudio obtenidos para las muestras de gasolina analizadas, pueden obtenerse las siguientes conclusiones:

1) Todas las muestras presentan un contenido de azufre dentro de los límites permitidos por la legislación en cada momento; $150 \mathrm{mg} / \mathrm{kg}$ para el segundo semestre del 2004 de y $50 \mathrm{mg} / \mathrm{kg}$ para el primer semestre del 2005.

2) Los responsables de la distribución han adecuado de forma eficaz los procesos de desulfuración aplicable a gasolinas con el objetivo de cumplir con los nuevos requisitos establecidos por el Real Decreto 1700/2003 en cuanto al contenido de azufre presente en ellas.

3) La gasolina de 98 octanos es la que presenta un menor contenido de azufre durante todo el periodo de estudio; tanto en el semestre correspondiente al año 2004 como al semestre correspondiente al año 2005.

4) Se ha constatado la rápida introducción en el mercado de gasolina de menor contenido de azufre, cumpliendo así con lo establecido en el Real Decreto 1700/2003 con en el apartado b) de su artículo 1 referente a la disponibilidad en el mercado nacional de gasolinas cuyo contenido de azufre sea inferior a $10 \mathrm{mg} / \mathrm{kg}$.

5) La formulación vigente de la gasolina comercializada en Galicia, cumple con la actual legislación de acuerdo con el contenido máximo de azufre.

6) El método empleado en el análisis demostró ser fiable a pesar de la dificultad que conlleva trabajar con muestras de tan alta volatilidad, si bien es tedioso y requiere que el análisis aunque sencillo sea realizado por un operador cualificado. La paulatina y progresiva reducción en el contenido de azufre aconseja el uso de otros métodos con mejor límite de detección y menor interferencia del operador como es el caso de la espectrofotometría de fluorescencia ultravioleta (UNE EN-ISO 20846), aconsejado ya para la determinación de bajas concentraciones de azufre en gasolina y gasóleo.

\section{REFERENCIAS}

Ali, M.F. y otros 4 autores; Deep desulphurization of gasoline and diesel fuels using non-hydrogen consuming techniques, Fuel: 85(10-11), 1354-1363 (2006). 
ASTM D1552-03, Standards Test Method for Sulfur in Petroleum Products (High-Temperature Method), Annual Book of ASTM Standards, Vol. 05.01, 625-631, Philadelphia, United States (2006).

Babich, I.V. y J.A. Moulijn; Science and technology of novel processes for deep desulfurization of oil refinery streams: a review, Fuel: 82(6), 607-631 (2003).

Bailey, D. y G. Solomon; Pollution prevention at ports: clearing the air, Environmental Impact Assessment Review: 24(7-8), 749-774 (2004)

Blumberg, K., M. Walsh y C. Pera; Low-Sulfur Gasoline and Diesel: The Key to Lower Vehicle Emissions, The International Council on Clean Transportation (ICCT), Napa, California, May (2003).

Cofala, J. y otros 10 autores; Cost-effective control of SO2 emissions in Asia, Journal of Environmental Management: 72 (3), 149-161 (2004).

Costabile, F y I. Allegrini; A new approach to link transport emissions and air quality: An intelligent transport system based on the control of traffic air pollution, Environmental Modelling \& Software: 23(3), 258-267, (2008).

De Lasa, H. y otros dos autores; Catalytic desulfurization of gasoline via dehydrosulfidation, Industrial and Engineering Chemistry Research: 45(4), 1291-1299 (2006).

Directiva 98/70/CE, Directiva del Parlamento Europeo y del Consejo de 13 de octubre de 1998 relativa a la calidad de la gasolina y el gasóleo y por la que se modifica la Directiva 93/12/CEE del Consejo, Diario Oficial de la Unión Europea, 58-68, Bruselas, Bélgica (1998).

Directiva 2003/17/CE, Directiva del Parlamento Europeo y del Consejo de 3 de marzo de 2003 por la que se modifica la Directiva 98/70/CE relativa a la calidad de la gasolina y el gasóleo, Diario Oficial de la Unión Europea, 10-19, Bruselas, Bélgica (2003).

Gurjar, B.R. y otros cuatro autores; Evaluation of emissions and air quality in megacities, Atmospheric Environment: 42(7), 1593-1606 (2008).

Ito E. y J.A. Rob van Veen; On novel processes for removing sulphur from refinery streams, Catalysis Today: 116 (4), 446-460 (2006).

Landulfo, E. y otros cuatro autores; Air quality assessment using a multi-instrument approach and air quality indexing in an urban area, Atmospheric Research: 85(1), 98-111 (2007).

Lumbreras, J. y otros tres autores; Assessment of vehicle emissions projections in Madrid (Spain) from 2004 to 2012 considering several control strategies, Transportation Research Part A, ISSN 0965-8564 (en línea), doi: 10.1016/j.tra.2008.01.026, 2008. http://www. sciencedirect.com. Acceso: 14 de Marzo (2008).

Özden, Ö, T. Dögeroglu y S. Kara; Assessment of ambient air quality in Eskisehir, Turkey, Environment Internacional, ISSN 0160-4120 (en línea), doi:10.1016/j.envint.2007.12.016, 2008. http://www.sciencedirect.com. Acceso: 11 de Marzo (2008).

R.D. 1700/2003, Real Decreto por el que se fijan las especificaciones de gasolinas, gasóleos, fuelóleos y gases licuados del petróleo, y el uso de biocarburantes, BOE No 307, 45961-45971, Madrid, España (2003).

Schifter, I. y otros cuatro autores; Impact of sulfur- in-gasoline on motor vehicle emissions in the metropolitan area of Mexico City. Fuel: 82(13), 1605-1612 (2003).

Siddiqui, M.A. y otros tres autores; Sulfur reduction in FCC gasoline using catalyst additives, Applied Catalysis A: General: 303(1), 116-120 (2006). 
UNE-EN ISO 3170, Productos petrolíferos líquidos. Toma de muestra manual, 1-38, Madrid, España (1999).

UNE-EN 14275, Combustibles para automoción. Evaluación de la calidad de la gasolina y del combustible para motores diésel (gasóleo). Toma de muestras en bombas y surtidores comerciales, 1-12, Madrid, España (2004).

Wang W. y otros tres autores; Desulfurization of gasolina by a new method of electrochemical catalytic oxidation, Fuel: 86(17-18), 2747-2753 (2007).

Yin, C. y D. Xia; A study of the distribution of sulfur compounds in gasolina produced in China. Part 3. Identification of individual sulfides and thiophenes, Fuel: 83(4-5), 433-441 (2004). 
